\title{
PEGylation-Dependent Metabolic Rewiring of Macrophages with Silk Fibroin Nanoparticles
}

\author{
John D. Totten, ${ }^{\dagger}$ Thidarat Wongpinyochit, ${ }^{\dagger}$ Joana Carrola, ${ }^{\ddagger}$ Iola F. Duarte, ${ }^{*}, \oplus$ and F. Philipp Seib ${ }^{*},, \S_{\odot}$ \\ ${ }^{\dagger}$ Strathclyde Institute of Pharmacy and Biomedical Sciences, University of Strathclyde, 161 Cathedral Street, Glasgow G4 ORE, U.K. \\ ${ }^{\ddagger}$ CICECO - Aveiro Institute of Materials, Department of Chemistry, University of Aveiro, 3810-193 Aveiro, Portugal \\ ${ }^{\S}$ Max Bergmann Center of Biomaterials Dresden, Leibniz Institute of Polymer Research Dresden, Hohe Strasse 6, 01069 Dresden, \\ Germany
}

\section{Supporting Information}

ABSTRACT: Silk fibroin nanoparticles are emerging as promising nanomedicines, but their full therapeutic potential is yet to be realized. These nanoparticles can be readily PEGylated to improve colloidal stability and to tune degradation and drug release profiles; however, the relationship between silk fibroin nanoparticle PEGylation and macrophage activation still requires elucidation. Here, we used in vitro assays and nuclear magnetic resonance based metabolomics to examine the inflammatory phenotype and metabolic profiles of macrophages following their exposure to unmodified or PEGylated silk fibroin nanoparticles. The macrophages internalized both types of nanoparticles, but they showed different phenotypic and metabolic responses to each nanoparticle type. Unmodified silk fibroin nanoparticles induced the upregulation of several processes, including production of proinflammatory mediators (e.g., cytokines), release of nitric oxide, and promotion of antioxidant activity. These responses were accompanied by changes in the macrophage metabolomic profiles that were consistent with a proinflammatory state and that indicated an increase in glycolysis and reprogramming of the tricarboxylic acid cycle and the creatine kinase/ phosphocreatine pathway. By contrast, PEGylated silk fibroin nanoparticles induced milder changes to both inflammatory and metabolic profiles, suggesting that immunomodulation of macrophages with silk fibroin nanoparticles is PEGylation-dependent. Overall, PEGylation of silk fibroin nanoparticles reduced the inflammatory and metabolic responses initiated by macrophages, and this observation could be used to guide the therapeutic applications of these nanoparticles.

KEYWORDS: silk, fibroin, silk nanoparticles, NMR metabolomics, macrophages

\section{INTRODUCTION}

The clinical approval of Abraxane in 2005 for the treatment of solid breast tumors was followed by a surge in the use of novel materials in the design of intravenous anticancer nanoparticles that target tumors. Drug-loaded nanoparticles can enter tumor tissues either passively, using the enhanced permeability and retention effect, ${ }^{1}$ or actively, using targeting moieties such as peptides, monoclonal antibodies, or aptamers. ${ }^{2,3}$ The physicochemical properties of nanoparticles therefore have a direct impact on their biological performance at the systemic, tissue, cellular, and subcellular levels. ${ }^{4,5}$ Factors such as the particle size, shape, and chemical composition dictate the likelihood of undesirable nanoparticle interactions with complement proteins and immunoglobulins in the circulating blood and with extracellular matrix proteins. These interactions then lead to rapid $(<1 \mathrm{~min})^{6}$ protein corona formation, opsonization of the particle, and clearance of the nanoparticles by professional macrophages of the mononuclear phagocytic system or by tumor-associated macrophages. ${ }^{7}$ This undesired clearance can be circumvented by conjugation of "stealth" polymers, such as poly(ethylene glycol) (PEG), to the nanoparticles. These types of conjugation have become standard practice to allow drugloaded nanoparticles to evade macrophage detection, thereby maximizing therapeutic payload delivery to the tumor site. ${ }^{8}$

Macrophages are versatile innate immune cells, and their processing of foreign materials (including nanosized drug carriers) plays an essential role in the initiation and coordination of inflammatory or anti-inflammatory immune responses. ${ }^{9}$ Until recently, the phagocytosis of nanomedicines was viewed as an undesirable event due to the risks of inducing drug hypersensitivity reactions. ${ }^{10}$ However, interest is now growing in the immunomodulatory potential of novel nanomaterials as immunological adjuvants that can influence sitespecific inflammation, particularly with regard to the tumor microenvironment. ${ }^{11}$ This has led to a revival in the demand for rigorous in vitro techniques that can be used to predict the

Received: November 2, 2018

Accepted: March 26, 2019 


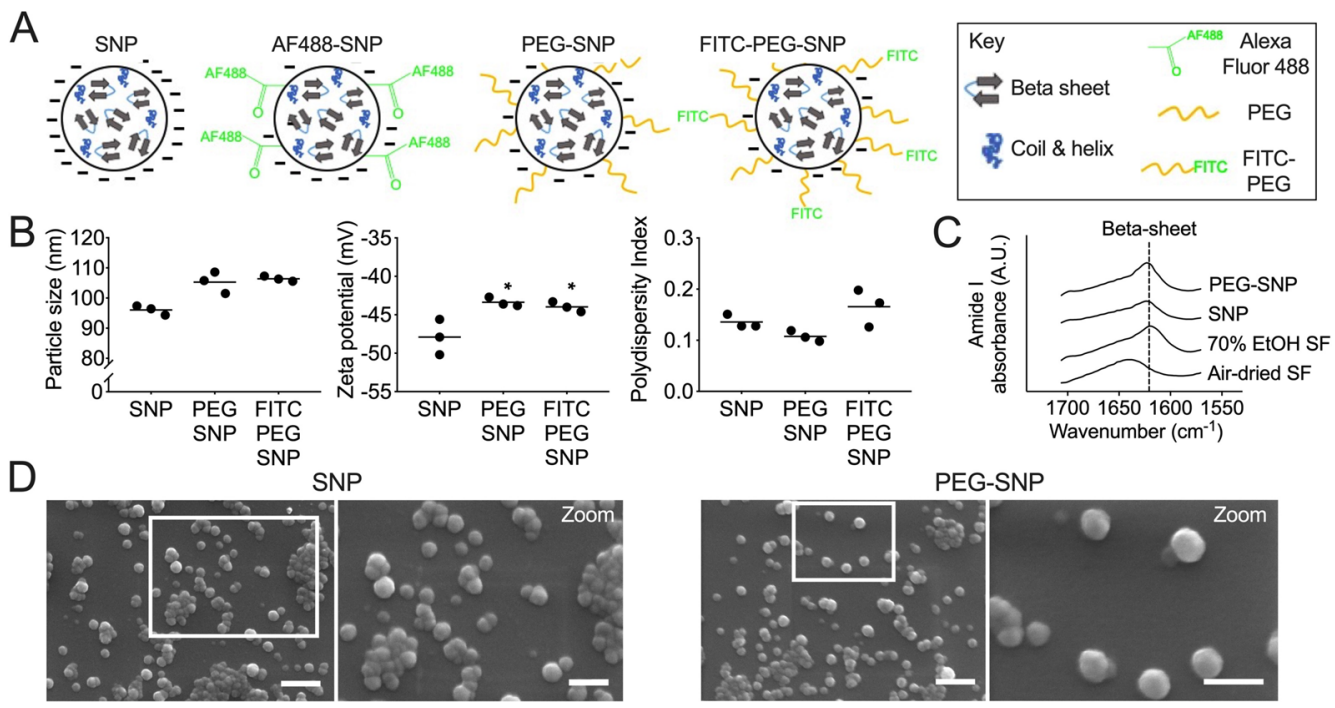

Figure 1. Characteristics of unmodified and PEGylated silk fibroin nanoparticles. (A) Schematic depicting unmodified (SNP), Alexa Fluor 488labeled (AF488-SNP), PEGylated (PEG-SNP), and FITC-PEGylated (FITC-PEG-SNP) silk fibroin nanoparticles used during the study. (B) Particle size distributions, $\zeta$ potentials, and polydispersity indices for unmodified, PEGylated, and FITC-PEGylated silk fibroin nanoparticles measured with DLS (*, $P<0.05$, one-way ANOVA) $(n=3)$. (C) FTIR absorbance spectra of unmodified and PEGylated SNPs presented alongside $70 \%$ ethanol treated or air-dried silk fibroin films (SF) as reference samples. The dotted lines at $1621 \mathrm{~cm}^{-1}$ indicate the $\beta$ sheet $(n=3)$. (D) Representative scanning electron images of unmodified and PEGylated silk fibroin nanoparticles (scale bars: left $=500 \mathrm{~nm}$; right $=250 \mathrm{~nm}$ ).

immunological outcome of novel materials being tested for advanced drug delivery applications. ${ }^{11}$

We recently proposed the use of NMR-based metabolomics to identify nanomedicine performance. NMR is a robust analytical technique that can be used to identify and quantify molecular structures such as cellular metabolites. ${ }^{12}$ Our previous comparative metabolomic assessment, which evaluated silk as a novel nanomaterial, revealed that unmodified silk fibroin nanoparticles performed in a similar manner to other emerging and clinically established nanomaterials, such as silica and poly(lactic-co-glycolic acid). Silk fibroin nanoparticles were able to drive metabolic reprogramming of RAW 264.7 macrophages toward induction of a proinflammatory M1-like metabolic profile. ${ }^{12}$

The design of silk nanoparticles for anticancer drug delivery has intensified over the past 5 years. For example, silk from the Bombyx mori cocoon is reverse-engineered into an aqueous silk fibroin solution using a process that removes sericin (i.e., a gumming agent present in the cocoon). ${ }^{13,14}$ The resulting silk fibroin solution is then desolvated to form spherical nanoparticles with an optimal $(\sim 100 \mathrm{~nm})$ size for intravenous administration. ${ }^{15,16}$ Silk fibroin nanoparticles can be readily decorated with PEG, ${ }^{17}$ and both physical and in vitro biological studies have validated the performances of unmodified and PEGylated silk fibroin nanoparticles for anticancer drug delivery applications. ${ }^{18-20}$ PEGylation significantly improves the colloidal stability while modulating the degradation and drug release profiles of silk fibroin nanoparticles ${ }^{17,18}$ but has no negative impacts on the ability of the nanoparticles to conduct lysosomotropic delivery of anticancer payloads. ${ }^{19}$ However, the immune adjuvanticity between unmodified and PEGylated silk fibroin nanoparticles has not yet been compared. Here, we examine the impacts of nanoparticle concentration and PEGylation on the phenotypic and metabolic reprogramming of macrophages induced by silk fibroin nanoparticles.

\section{EXPERIMENTAL SECTION}

Production and Characterization of Unmodified and PEGylated Silk Fibroin Nanoparticles. Silk fibroin was extracted as detailed previously. ${ }^{15}$ Briefly, Bombyx mori cocoons were cut into 5 $\times 5 \mathrm{~mm}$ pieces and degummed in $\mathrm{Na}_{2} \mathrm{CO}_{3}(0.02 \mathrm{M})$ for $60 \mathrm{~min}$ to quantitatively remove sericin. Fibers were rinsed with $\mathrm{ddH}_{2} \mathrm{O}$, airdried, and dissolved in $\mathrm{LiBr}$ solution $(9.3 \mathrm{M})$ at $60^{\circ} \mathrm{C}$, yielding a 25 wt $\%$ solution. This solution was dialyzed (molecular weight cutoff $3500 \mathrm{~g} / \mathrm{mol}$ ) against $\mathrm{ddH}_{2} \mathrm{O}$ for $72 \mathrm{~h}$ to remove the $\mathrm{LiBr}$ salt. The resulting aqueous silk fibroin solution was cleared by centrifugation (9500 $\times g$ for $20 \mathrm{~min}$ at $5{ }^{\circ} \mathrm{C}$ and repeated once), diluted to $5 \%(\mathrm{w} /$ $\mathrm{v}$ ), and added dropwise to acetone to produce spherical silk fibroin nanoparticles. Silk fibroin nanoparticles were surface-decorated with polyethylene glycol (PEG) using a method that has been reported previously, ${ }^{17}$ and a visual format of silk fibroin nanoparticle production is available. ${ }^{15}$ All samples were washed thoroughly and stored at $4{ }^{\circ} \mathrm{C}$ for up to 4 weeks.

A Zetasizer Nano-ZS (Malvern Panalytical, Worcestershire, U.K.) was used to characterize the respective particle size distributions, $\zeta$ potentials, and polydispersity indices (PDI) of unmodified and PEGylated silk fibroin nanoparticles using dynamic light scattering (DLS) in $\mathrm{ddH}_{2} \mathrm{O}$. A refractive index of 1.33 was used for $\mathrm{ddH}_{2} \mathrm{O}$. For phenotypic and metabolic analyses, silk fibroin nanoparticles were either left in their unmodified form or surface-decorated with unlabeled PEG at a $1: 1 \mathrm{w} / \mathrm{v}$ ratio (as detailed above). For fluorescence analyses, unmodified silk fibroin nanoparticles were either labeled directly with Alexa Fluor 488 or PEGylated with a 1:4 ratio of FITC-PEG and unlabeled PEG, as described below. A schematic of each formulation is shown in Figure 1A.

Fluorescence Labeling of Unmodified and PEGylated Silk Fibroin Nanoparticles. Unmodified silk fibroin nanoparticles (1 $\mathrm{mL}, 10 \mathrm{mg} / \mathrm{mL})$ were resuspended in $\mathrm{NaHCO}_{3}(1 \mathrm{~mL}, 0.2 \mathrm{M}, \mathrm{pH}$ 8.6), and Alexa Fluor 488 succinimidyl ester $(100 \mu \mathrm{L}, 1 \mathrm{mg} / \mathrm{mL})$ (Thermo Fisher Scientific, Waltham, MA, USA) was added as detailed previously. ${ }^{17}$ Fluorescent PEGylated silk fibroin nanoparticles were produced by resuspending unmodified silk fibroin nanoparticles (1 $\mathrm{mL}, 10 \mathrm{mg} / \mathrm{mL})$ in $\mathrm{NaHCO}_{3}(1 \mathrm{~mL}, 0.2 \mathrm{M}, \mathrm{pH} 8.6)$ and adding fluorescein PEG succinimidyl ester $(250 \mu \mathrm{L}, 30 \mathrm{mg} / \mathrm{mL}$ in DMSO) (FITC-PEG-NHS, MW $5000 \mathrm{~g} / \mathrm{mol}$, Nanocs, Boston, MA, USA). The sample was incubated for $4 \mathrm{~h}$ at room temperature in the dark with stirring, and then TST-activated mPEG $(750 \mu \mathrm{L}, 30 \mathrm{mg} / \mathrm{mL})$ 
( $5000 \mathrm{~g} / \mathrm{mol}$, Sigma-Aldrich, St. Louis, MO, USA) in $\mathrm{NaHCO}_{3}(0.2$ $\mathrm{M}, \mathrm{pH}$ 8.6) was added and reacted overnight at $4{ }^{\circ} \mathrm{C}$ with stirring. The fluorescently labeled unmodified and PEGylated silk fibroin nanoparticles were then centrifuged $(10000 \times g$ for $45 \mathrm{~min})$, and the free (unconjugated) dye was removed by washing the pellet in acidified water ( $\mathrm{pH} 4.6)$. This was repeated once more with acidified water and then three more times with ultrapure water. All fluorescent nanoparticles were refrigerated $\left(4^{\circ} \mathrm{C}\right)$ and protected from light until use.

Cell Culture. RAW 264.7 cells are a mouse macrophage cell line originally extracted from ascites of an Abelson murine leukemia virusinduced tumor and thought to be derived from peritoneal monocytes. ${ }^{21}$ The culture of RAW 264.7 cells in complete medium has been described previously. ${ }^{12}$ Subconfluent flasks were passaged by scraping cells, centrifuging at $380 \times g$ for $4 \mathrm{~min}$, and replating them at ratios of 1:5-1:10 onto plasma-treated tissue culture polystyrene. The cell passage number was controlled during the study, and all experiments were conducted using cells under passage 20. Seeding for polarization experiments was conducted at a density of $1.5 \times 10^{4}$ cells $/ \mathrm{cm}^{2}$, and cells were allowed to recover for $24 \mathrm{~h}$ prior to conducting the in vitro experiments.

Scanning Electron Microscopy (SEM). Unmodified or PEGylated silk fibroin nanoparticles $(1 \mathrm{mg} / \mathrm{mL})$ in distilled water were lyophilized and sputter-coated with gold prior to imaging with 40000 -fold magnification at $5 \mathrm{kV}$ on a Hitachi SU6600 SEM, as detailed previously. ${ }^{17}$

RAW 264.7 cells were seeded as detailed above onto glass coverslips recovered for $24 \mathrm{~h}$. They were then dosed for $24 \mathrm{~h}$ with unmodified or PEGylated silk fibroin nanoparticles (detailed below). Next cells were fixed ( $2 \% \mathrm{v} / \mathrm{v}$ glutaraldehyde in phosphate-buffered saline (PBS)), washed with ultrapure water, dehydrated, and dried at the critical point (EM CPD300, Leica Microsystems, Wetzlar, Germany). Samples were sputter-coated with gold and imaged by SEM with 2000-fold magnification at $5 \mathrm{kV}$.

Fourier Transform Infrared Spectroscopy (FTIR). The secondary silk structures were determined for air-dried silk films, $70 \%$ ethanol treated silk films, and lyophilized unmodified and PEGylated silk nanoparticles using Fourier transform infrared (FTIR) spectroscopy (TENSOR II FTIR spectrometer, Bruker Optik GmbH, Ettlingen, Germany), as detailed elsewhere. ${ }^{17}$ Briefly, the samples were subjected to 128 scans at a $4 \mathrm{~cm}^{-1}$ resolution over the wavenumber range of 400 to $4000 \mathrm{~cm}^{-1}$. The amide I region was identified: $1605-1615 \mathrm{~cm}^{-1}$ as side chain/aggregated strands, 16161637 and $1697-1703 \mathrm{~cm}^{-1}$ as a $\beta$-sheet structure, $1638-1655 \mathrm{~cm}^{-1}$ as a random coil structure, $1656-1662 \mathrm{~cm}^{-1}$ as $\alpha$-helical bands, and $1663-1696 \mathrm{~cm}^{-1}$ as turns. ${ }^{22}$

Endocytosis of Unmodified and PEGylated Silk Fibroin Nanoparticles. RAW 264.7 cells were seeded and cultured as described above but in a complete DMEM medium without phenol red. Fluorescently labeled silk nanoparticles were used in this study, and cells were either left untreated or dosed with unmodified or PEGylated silk fibroin nanoparticles at either 0.1 or $0.5 \mathrm{mg} / \mathrm{mL}$. Cells were incubated for $24 \mathrm{~h}$ in total with LysoTracker Red ( $50 \mathrm{nM}$ in complete growth medium) (Thermo Scientific, Waltham, MA, USA) added in the final $2 \mathrm{~h}$ of the experiment. Cells were then placed on ice, washed three times with ice cold PBS, stained with Hoechst $33342(1 \mu \mathrm{g} / \mathrm{mL}$ in serum free medium $)$ and live-imaged immediately using a Leica TCS-SP5 confocal microscope as detailed previously. ${ }^{19}$

Calculation of Colocalization Coefficients. Colocalization between nanoparticles and lysosomes was determined on representative confocal images using the JACoP ImageJ plug-in to calculate Mander's overlap coefficients for green (nanoparticle) and magenta (lysosome) channels. Mander's coefficient ranges from 0 to 1 and indicates the degree of colocalization between two channels. ${ }^{23,24}$ The default JACoP algorithm was used on threshold adjusted images to calculate Mander's coefficients for each treatment group.

MTT Assay. The cytotoxicity of unmodified and PEGylated silk fibroin nanoparticles was verified using 3-(4,5-dimethylthiazol-2-yl)2,5-diphenyltetrazolium bromide (MTT) assays. ${ }^{25}$ After seeding and recovery (detailed above), cells were either left untreated or dosed for
$24 \mathrm{~h}$ in complete DMEM supplemented with (i) $1 \% \mathrm{v} / \mathrm{v}$ Triton X100 , (ii) $0-1 \mathrm{mg} / \mathrm{mL}$ unmodified silk fibroin nanoparticles, (iii) $0-1$ $\mathrm{mg} / \mathrm{mL}$ PEGylated silk fibroin nanoparticles, or (iv) PEG (5000 g/ mol; Iris Biotech GmbH, Marktredwitz, Germany). MTT ( $5 \mathrm{mg} / \mathrm{mL}$ in PBS) was then added at $10 \%$ of the volume of cell culture media, and cells were cultured again at $37^{\circ} \mathrm{C}$ for $5 \mathrm{~h}$. The medium was then aspirated, the formazan product was solubilized with DMSO (100 $\mu \mathrm{L}$ ), and the absorbance was measured at $570 \mathrm{~nm}$. All data sets were normalized to the Triton X-100 positive control (Figure S1) to determine the percentage of cell viability following each treatment.

Phenotypic Response to Unmodified and PEGylated Silk Fibroin Nanoparticles. RAW 264.7 cells were seeded and allowed to recover for $24 \mathrm{~h}$ as detailed above. Next, cells were dosed for $24 \mathrm{~h}$ in complete medium supplemented with a low $(0.1 \mathrm{mg} / \mathrm{mL})$ or high $(0.5 \mathrm{mg} / \mathrm{mL})$ concentration of either unmodified or PEGylated silk fibroin nanoparticles (note that macrophages were exposed to silk fibroin nanoparticles without prior priming). A $1 \% \mathrm{v} / \mathrm{v}$ Triton X-100 positive control sample was included to assess plasma membrane integrity. However, for tumor necrosis factor alpha (TNF- $\alpha$ ), nitric oxide $\left(\mathrm{NO}_{2}^{-}\right)$production, antioxidant capacity, and reactive oxygen species, a positive control using lipopolysaccharide $(1.5 \mathrm{ng} / \mathrm{mL})$ was included instead to provide baseline phenotypic information on RAW 264.7 cells in an activated state. Following the $24 \mathrm{~h}$ incubation period, the growth medium was collected, clarified by centrifugation $(380 \times g$ for $10 \mathrm{~min}$ ), transferred to $2 \mathrm{~mL}$ low-protein-binding Eppendorf tubes, and stored at $-80{ }^{\circ} \mathrm{C}$. The conditioned growth medium samples were assayed to quantitatively monitor plasma membrane integrity (lactate dehydrogenase assay), TNF- $\alpha$ (TNF- $\alpha$ ELISA), and nitric oxide levels (Griess assay). Cell lysates were generated as detailed below to measure antioxidant capacity and reactive oxygen species.

The lactate dehydrogenase assay was performed with a Pierce LDH Cytotoxicity Assay Kit (Thermo Fisher Scientific, Waltham, MA, USA) following the manufacturer's instructions to assess membrane integrity in positive and negative controls, nanoparticle, and LPS treated cells.

A mouse TNF- $\alpha$ DuoSet ELISA kit (R\&D Systems, Minneapolis, MN, USA) was used to quantify TNF- $\alpha$ levels in a conditioned culture medium. Nitric oxide was measured with the Griess assay as detailed elsewhere. ${ }^{26,27}$

Antioxidant Assay. Cells were treated as detailed above. Next, cells were washed three times with $1 \times$ Hank's balanced salt solution (HBSS), scraped and collected into falcon tubes, and centrifuged at $2000 \times g$ for $10 \mathrm{~min}$ at $4{ }^{\circ} \mathrm{C}$. The supernatant was decanted, and the pellet was resuspended in $1 \mathrm{~mL}$ HBSS and sonicated on ice. Lysates were then centrifuged at $10000 \times g$ for $15 \mathrm{~min}$ at $4{ }^{\circ} \mathrm{C}$, and the supernatant was collected and stored at $-80{ }^{\circ} \mathrm{C}$. Total antioxidant capacity was quantified by analyzing supernatant samples with an antioxidant assay kit (Cayman Chemical, MI, USA).

Reactive Oxygen Species Assay. Cells were treated as detailed above. Next, cells were washed three times with $1 \times$ HBSS, stained for $1 \mathrm{~h}$ with CellROX Deep Red Reagent (Thermo Fisher Scientific, Waltham, MA, USA), scraped into $3 \mathrm{~mL}$ falcon tubes, and stained with SYTOX Green (ThermoFisherScientific, Waltham, MA, U.S.A.) as an exclusion dye. Cells were analyzed immediately on a BD FACSCanto, with 20000 live events being recorded per sample. Flow cytometry data was subsequently analyzed with FlowJo (FlowJo, LLC, Ashland, OR, USA).

Cytokine Profile. A mouse cytokine proteome profiler (Panel A, R\&D Systems, Minneapolis, MN, USA) was used, according to the manufacturer's instructions. Blots from the arrays were exposed to UltraCruz autoradiography film (Santa Cruz Biotech Inc., Dallas, TX, USA), digitized (18 MP Canon EOS100D, Canon Inc., Tokyo, Japan), threshold adjusted, and analyzed using Image J v1.51k 1 (National Institutes of Health, Bethesda, MD, USA).

Metabolomic Sample Preparation. RAW 264.7 cells were dosed for $24 \mathrm{~h}$ with unmodified or PEGylated silk fibroin nanoparticles at either 0.1 or $0.5 \mathrm{mg} / \mathrm{mL}$. Next, the culture medium was collected, clarified by centrifugation $(380 \times g$ for $10 \mathrm{~min})$, lyophilized, and stored at $-80{ }^{\circ} \mathrm{C}$ until analysis. The protocol for 


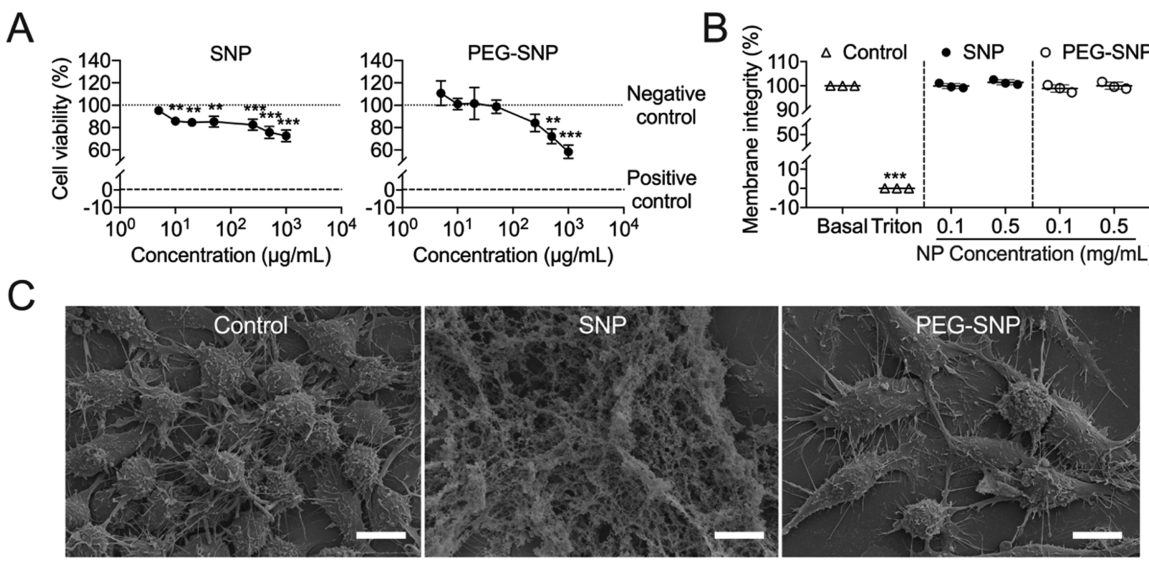

Figure 2. Macrophage response toward unmodified and PEGylated silk fibroin nanoparticles. (A) In vitro cytotoxicity of unmodified (SNP) and PEGylated (PEG-SNP) silk fibroin nanoparticles following a $24 \mathrm{~h}$ incubation in complete media supplemented with $0-1000 \mu \mathrm{g} / \mathrm{mL}$ nanoparticles $(*, P<0.05 ; * *, P<0.01$; ***, $P<0.001$, one-way ANOVA between control and nanoparticle treated groups; $n=3)$. (B) Membrane integrity of RAW 264.7 cells following a $24 \mathrm{~h}$ incubation in complete media supplemented with 0.1 or $0.5 \mathrm{mg} / \mathrm{mL}$ unmodified (SNP) or PEGylated (PEGSNP) silk fibroin nanoparticles (*, $P<0.05$; **, $P<0.01$; ***, $P<0.001$, one-way ANOVA untreated and nanoparticle treated groups; $n=3$ ). (C) Scanning electron microscope images of RAW 264.7 cells following a $24 \mathrm{~h}$ dose with $0.1 \mathrm{mg} / \mathrm{mL}$ unmodified (SNP) or PEGylated (PEG-SNP) silk fibroin nanoparticles. Scale bar $=100 \mu \mathrm{m}$.

metabolomic sample preparation of cell extracts is outlined in detail in our previous report. ${ }^{12}$

NMR Spectroscopy. At the time of NMR analysis, dried samples of cell culture media and aqueous cell extracts were reconstituted in deuterated phosphate buffer $(600 \mu \mathrm{L}, 100 \mathrm{mM}, \mathrm{pH} 7.4)$ containing 3(trimethylsilyl)-1-propanesulfonic acid- $d_{6}$ sodium salt (TSP) (0.1 $\mathrm{mM}$ ), whereas lipophilic cell extracts were reconstituted in deuterated chloroform containing $0.03 \%$ tetramethylsilane (TMS). For NMR analysis, $550 \mu \mathrm{L}$ of each sample was transferred into $5 \mathrm{~mm}$ NMR tubes. NMR spectra were acquired on a Bruker Avance DRX-500 spectrometer operating at $500.13 \mathrm{MHz}$ for ${ }^{1} \mathrm{H}$ observation, at $298 \mathrm{~K}$, using a $5 \mathrm{~mm}$ probe. Standard $1 \mathrm{D}{ }^{1} \mathrm{H}$ spectra (pulse programs "noesyprld", with water suppression, for medium samples/aqueous extracts, and "zg" for lipophilic extracts) were recorded with a 7002.8 $\mathrm{Hz}$ spectral width, $32 \mathrm{k}$ data points, a $2 \mathrm{~s}$ relaxation delay, and 512 scans. Spectral processing comprised exponential multiplication with $0.3 \mathrm{~Hz}$ line broadening, zero filling to $64 \mathrm{k}$ data points, manual phasing, baseline correction, and chemical shift calibration to the TSP or TMS signal at $0 \mathrm{ppm}$. Metabolites were identified based on 2D spectra and consultation of spectral reference databases, as detailed previously. $^{12}$

Integration of NMR Spectra and Multivariate Quantification. NMR spectra were normalized by total spectral area (excluding the region comprising the suppressed water signal) to eliminate potential difference due to cell number variations. Principal component analysis and partial least squares discriminant analysis were then applied using SIMCA-P 11.5 software (Umetrics, Umeå, Sweden), with a default 7-fold internal cross validation, from which $Q^{2}$ and $R^{2}$ values, reflecting predictive capability and explained variance, respectively, were extracted.

Metabolite variations were quantified by normalizing selected signals in the 1D spectra to total spectral area. For each metabolite, the percentage variation in nanoparticle-exposed samples was calculated relative to controls, together with the effect size adjusted for small sample numbers and the respective standard errors. ${ }^{28}$ The metabolite variations of large magnitude (i.e., with an absolute effect size $\geq 0.8)^{28}$ were plotted as a heat map.

Data and Statistical Analyses. Data was analyzed (Figures 1-4) using GraphPad Prism 7.0a (GraphPad Software, La Jolla, CA). Student's $t$ tests were used to analyze sample pairs. One-way analysis of variance (ANOVA) between basal and nanoparticle treated groups was conducted followed by Bonferroni's multiple comparison post hoc test for multiple samples. Statistical significance is indicated by asterisks in each figure legend. All data are plotted as mean \pm standard deviation and, unless otherwise stated, refer to a minimum of three
A

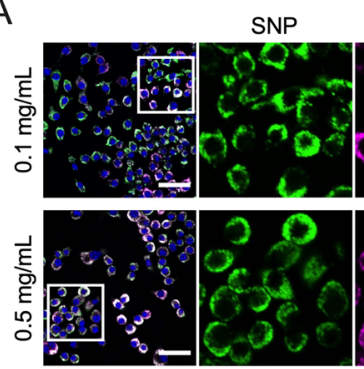

B

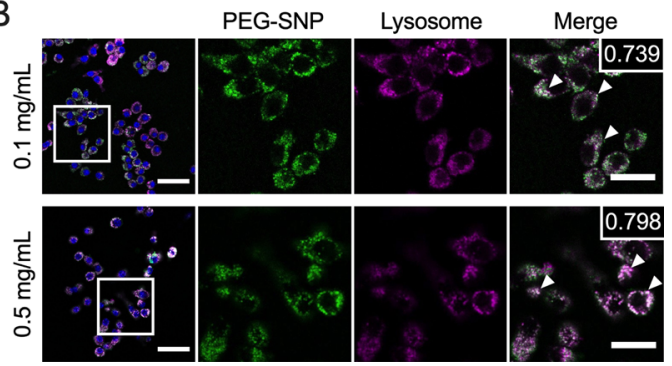

Figure 3. Cellular uptake of unmodified and PEGylated silk fibroin nanoparticles. Live RAW 264.7 macrophages following a $24 \mathrm{~h}$ dose of (A) unmodified or (B) PEGylated silk fibroin nanoparticles at concentrations of 0.1 or $0.5 \mathrm{mg} / \mathrm{mL}$. (Scale bars: left $=40 \mu \mathrm{m}$; right (zoomed) $=20 \mu \mathrm{m}$ ). Data acquisition using confocal microscopy. Numbers on merged images show Mander's coefficients calculated for each treatment group. Arrowheads identify colocalization between the nanoparticle-associated and lysosomal signals.

independent biological repeats. Statistical analysis of NMR data has been described above.

\section{RESULTS}

Characterization of Unmodified and PEGylated Silk Fibroin Nanoparticles. DLS was used to quantitatively verify unmodified and PEGylated silk fibroin nanoparticle sizes, $\zeta$ potentials, and polydispersity indices of each preparation using different batches (Figure 1). An increase in particle size was noted between the unmodified silk fibroin nanoparticles (96.0 $\mathrm{nm}$ ) and the PEGylated and FITC-PEGylated silk fibroin nanoparticles (105.3 and $106.4 \mathrm{~nm}$, respectively). The $\zeta$ 

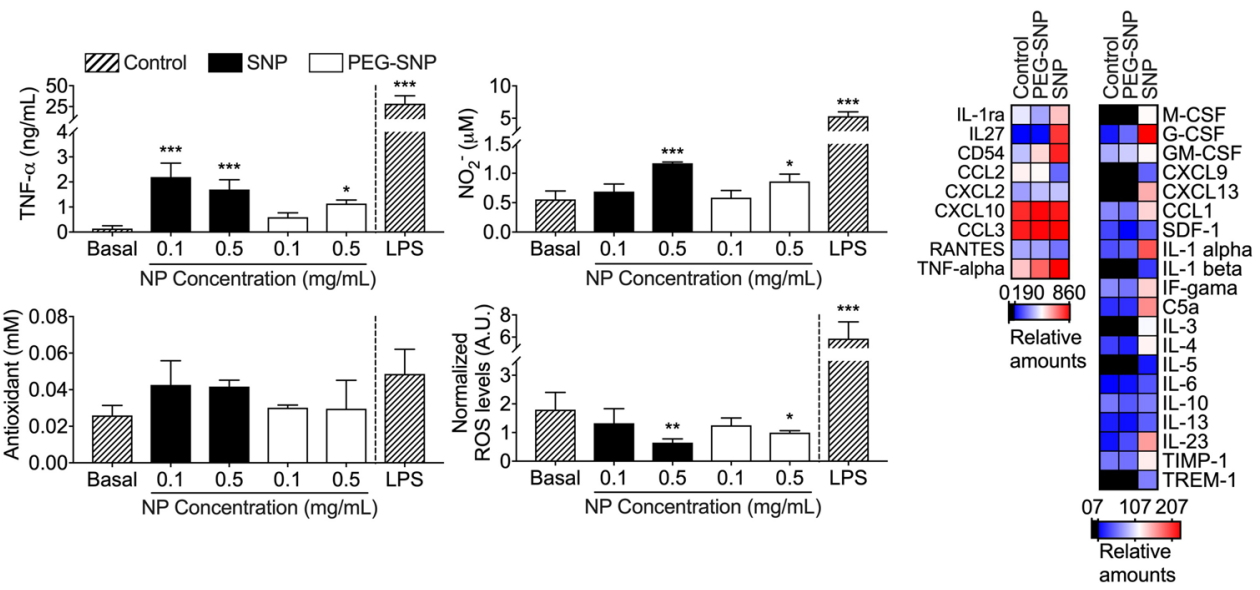

Figure 4. Phenotypic changes induced in macrophages exposed to unmodified (SNP) and PEGylated (PEG-SNP) silk fibroin nanoparticles. (A) TNF- $\alpha$ release, (B) nitric oxide $\left(\mathrm{NO}_{2}^{-}\right)$levels, (C) total antioxidant capacity, and (D) reactive oxygen species (ROS) from RAW 264.7 macrophages following a $24 \mathrm{~h}$ incubation in complete media supplemented with either unmodified or PEGylated silk fibroin nanoparticles; $1.5 \mathrm{ng} /$ $\mathrm{mL}$ lipopolysaccharide (LPS) served as a positive control (*, $P<0.05$; **, $P<0.01$; ***, $P<0.001$, A, B, and C: $n=3$, D: $n=4$ ). (E) Cytokine profile of RAW 264.7 cells treated with $0.5 \mathrm{mg} / \mathrm{mL}$ nanoparticles; untreated cells served as the control.

potential was also significantly influenced by the PEGylation process. In their unmodified state, the silk fibroin nanoparticles had a $\zeta$ potential of $-47.9 \mathrm{mV}$. However, this increased to -43.4 and $-44.0 \mathrm{mV}$ for PEGylated and FITC-PEGylated silk fibroin nanoparticles, respectively. All three silk fibroin nanoparticle types showed a narrow polydispersity (Figure 1B). The FTIR absorbance analyses at $1621 \mathrm{~cm}^{-1}$ confirmed a high abundance of $\beta$ sheets within the structures of all three nanoparticle formulations when compared to ethanol-treated silk film controls (Figure 1C). Quantitative measurements were supported by SEM images that confirmed the spherical shape of the unmodified and PEGylated silk fibroin nanoparticles (Figure 1D).

Interactions of Unmodified and PEGylated Silk Fibroin Nanoparticles with Macrophages. RAW 264.7 cells were selected as a model mouse macrophage cell line to allow for continuity with our initial study. ${ }^{12}$ A combination of biochemical in vitro assays and NMR metabolomics was used to elucidate silk-nanoparticle-induced shifts in phenotypic and metabolic profiles. First, the cytotoxicity of silk fibroin nanoparticles was assessed. Exposure to unmodified silk fibroin nanoparticles caused a significant reduction in cell viability when compared to untreated controls. However, statistical analysis between cells exposed to unmodified or PEGylated silk fibroin nanoparticles at equivalent concentrations revealed no significant differences in cell viability. The unmodified and PEGylated silk fibroin nanoparticles showed cytotoxicities of less than $15 \%$ at exposure levels of up to $100 \mu \mathrm{g} / \mathrm{mL}$ (Figure 2A). At higher concentrations, both particle types reduced cell viability. At the maximum tested concentration, the cell viability was reduced more substantially for the PEGylated silk fibroin nanoparticles than for the unmodified ones; however, the $\mathrm{IC}_{50}$ value remained $>1000 \mu \mathrm{g} / \mathrm{mL}$ (Figure $2 \mathrm{~A}$ ). Parallel experiments that tested the cytotoxicity of water-soluble PEG revealed no significant changes in cell viability over the tested concentration range (Figure S2). The macrophage plasma membrane integrity was monitored by lactate dehydrogenase leakage into the culture medium. Exposure of the macrophages to unmodified or PEGylated silk fibroin nanoparticles at either low $(0.1 \mathrm{mg} / \mathrm{mL})$ or high $(0.5 \mathrm{mg} / \mathrm{mL})$ nanoparticle concentrations caused no leakage of lactate dehydrogenase, indicating no plasma membrane damage (Figure 2B). Qualitative assessment by SEM showed no gross morphological changes of the macrophages following exposure to the nanoparticles. SEM images of RAW 264.7 macrophages treated at the low nanoparticle concentration showed that the unmodified nanoparticles formed aggregates at the cell surface, whereas the PEGylated silk fibroin nanoparticles did not (Figure 2C).

We also verified whether endocytic uptake might be involved in the observed effects by studying the intracellular fate of the unmodified and PEGylated silk nanoparticles by live-cell confocal microscopy. We used LysoTracker Red to label the RAW 264.7 cells, and we observed nanoparticle accumulation within lysosomes at $24 \mathrm{~h}$, as evidenced by the substantial colocalization of the silk fibroin nanoparticles with the lysosomal signals. This colocalization occurred regardless of particle PEGylation or concentration, as evidenced by similar Mander's coefficients (numbers on the merged images in Figure 3). However, the unmodified silk nanoparticles showed some evidence of aggregation at the cell membrane. This aggregation was reduced, and the lysosomes were smaller in the macrophages dosed with PEGylated silk fibroin nanoparticles.

Inflammatory Response to Unmodified and PEGylated Silk Nanoparticles. Next, the inflammatory phenotype of RAW 264.7 macrophages in response to low and high nanoparticle concentrations was determined. Cell exposure to unmodified silk fibroin nanoparticles induced significant TNF- $\alpha$ release at both nanoparticle concentrations (2.20 and $1.70 \mathrm{ng} / \mathrm{mL}$, respectively) when compared to basal levels $(0.14 \mathrm{ng} / \mathrm{mL})$ (Figure $4 \mathrm{~A})$. A milder TNF- $\alpha$ response was observed when macrophages were treated with PEGylated silk fibroin nanoparticles, with only the high concentration causing a significant increase in TNF- $\alpha$ release $(1.14 \mathrm{ng} / \mathrm{mL})$. When assessing nitric oxide levels and reactive oxygen species, neither factor was influenced by a low concentration of either unmodified or PEGylated silk fibroin nanoparticles (Figure $4 \mathrm{~B}, \mathrm{D})$. However, at the high nanoparticle concentration, unmodified silk fibroin nanoparticles significantly increased nitric oxide levels (111\%) yet decreased reactive oxygen species levels by $64 \%$. A similar trend was observed with the 
high PEGylated silk fibroin nanoparticle concentration (55\% increase of nitric oxide levels and $44 \%$ drop in reactive oxygen species). Total antioxidant capacity in RAW 264.7 increased substantially compared to basal levels following treatment with low and high concentrations of unmodified silk fibroin nanoparticles. However, only minimal changes were observed following treatment with PEGylated silk fibroin nanoparticles (Figure 4C).

The cell culture medium was also assayed to further evaluate the cytokine response of RAW 264.7 cells following nanoparticle exposure (Figure $4 \mathrm{E}$ ). In addition to promoting a marked increase in the expression of TNF- $\alpha$, the unmodified silk fibroin nanoparticles upregulated the expression of several colony stimulating factors (M-CSF, G-CSF, and GM-CSF), chemokines (CCL1, CXCL 9, CXCL 13), and other essential proinflammatory cytokines (IFN- $\gamma$, IL- $1 \alpha$, IL-23, and IL-27). By contrast, PEGylated silk fibroin nanoparticle treatment caused a milder (or negligible) response in proinflammatory cytokine expression when compared to unmodified silk fibroin nanoparticles. The anti-inflammatory cytokine IL-10 remained unchanged regardless of the type of silk fibroin nanoparticle used, whereas IL-1ra expression increased in response to unmodified silk fibroin nanoparticles yet decreased following a dose with PEGylated silk fibroin nanoparticles.

Metabolomic Response to Unmodified and PEGylated Silk Nanoparticles. Next, the metabolic profiles of RAW 264.7 macrophages were analyzed to further characterize the effects of silk fibroin nanoparticle PEGylation and concentration on inflammatory modulation. A score scatter plot (Figure 5A) produced by applying principal component analysis to the ${ }^{1} \mathrm{H}$ NMR spectra of aqueous cell extracts showed a separation between control and nanoparticle treated samples. Samples clustered differently depending on

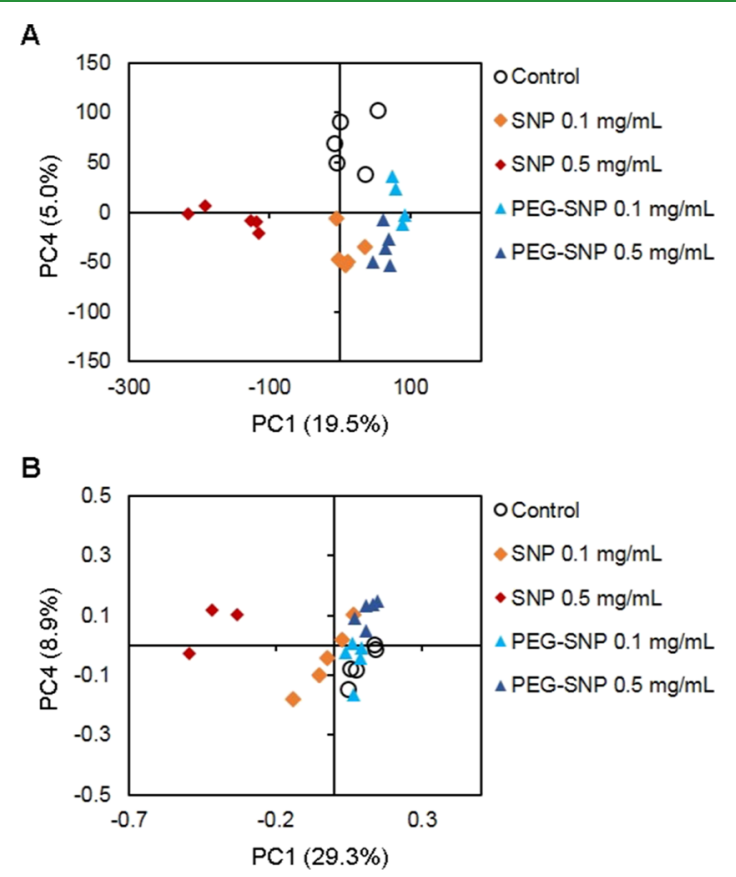

Figure 5. Score scatter plots obtained by principal component analysis of ${ }^{1} \mathrm{H}$ NMR spectra from (A) aqueous extracts and (B) lipophilic extracts of RAW 264.7 macrophages treated for $24 \mathrm{~h}$ with unmodified or PEGylated silk fibroin nanoparticles (SNPs) at concentrations of either 0.1 or $0.5 \mathrm{mg} / \mathrm{mL}$. nanoparticle treatment, and in particular the high concentration of unmodified silk nanoparticles separated clearly from the remaining samples along principal component 1 (PC1, explaining $19.5 \%$ of total variance). These results were complemented by principal component analysis of lipophilic cell extracts (Figure 5B), which showed some overlap between sample groups, especially in the case of PEGylated particles as they barely separated from controls. By contrast, different clusters were observed for lipophilic samples obtained from cells treated with unmodified silk fibroin nanoparticles, with the $0.5 \mathrm{mg} / \mathrm{mL}$ cluster grouping separately from control clusters.

The principal component analysis score map (Figure 6A) obtained for samples from macrophages treated with

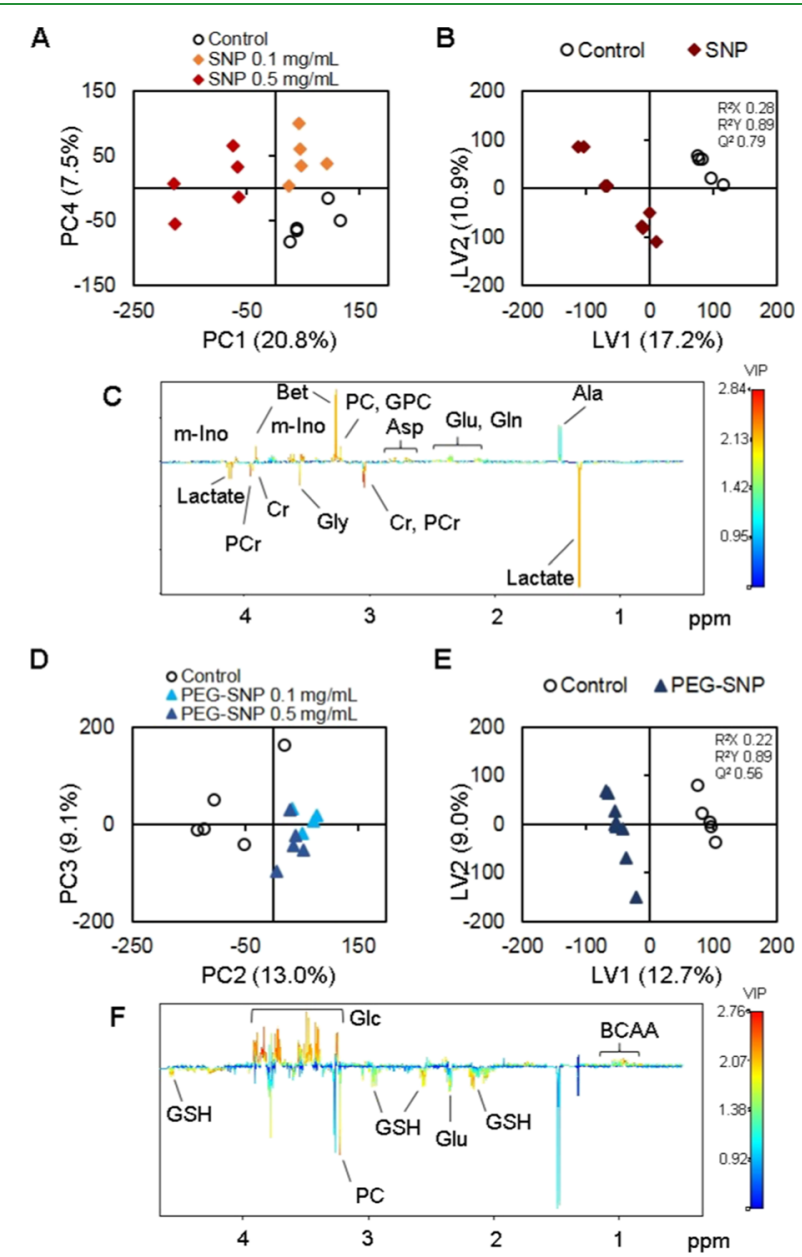

Figure 6. Multivariate analysis of ${ }^{1} \mathrm{H}$ NMR spectra from aqueous extracts of control macrophages and macrophages exposed for $24 \mathrm{~h}$ to (A-C) unmodified (SNP) and (D-F) PEGylated (PEG-SNP) silk fibroin nanoparticles: (A,D) principal component analysis score scatter plots; (B,E) partial least squares discriminant analysis score scatter plots generated by pairwise comparisons (control and (PEG)SNP, 0.1 and $0.5 \mathrm{mg} / \mathrm{mL}$ as one class); (C,F) loadings $w$ of latent variable 1 (LV1), colored as a function of variable importance to the projection (VIP).

unmodified silk fibroin nanoparticles confirmed the separation between control, low dose, and high dose samples. Partial least squares discriminant analysis (Figure 6B) further revealed robust discrimination between control and nanoparticletreated cells (Q2 0.79) due to differences in the levels of metabolites highlighted in the corresponding loading plots 
(Figure 6C). In particular, silk fibroin nanoparticle treated cells (negative scores in first latent variable, LV1) were characterized mainly by higher intracellular levels of lactate, creatine, phosphocreatine, and glycine (signals with negative LV1 loadings), together with lower levels of a few amino acids, choline compounds, betaine, and myo-inositol (positive loadings). In samples from macrophages treated with PEGylated silk fibroin nanoparticles (Figure 6D), the low and high dose samples largely overlapped in the principal component analysis score scatter plot, suggesting little dependence on nanoparticle concentration. When considering the two exposure concentrations within a single class, nanoparticle treated samples strongly differed from the controls by partial least squares discriminant analysis (Figure $6 \mathrm{E}$ ). The corresponding loading profiles (Figure 6F) suggested decreased levels of branched chain amino acids and glucose, along with increased levels of glutamate, glutathione, and phosphocholine, in treated cells compared with controls.

Spectral integration was then employed to confirm and extend the analysis of quantitative variations in individual metabolites (summarized in Figure 7). Overall, silk fibroin nanoparticles caused changes in 28 intracellular polar metabolites associated with different metabolic pathways. When measuring glycolytic metabolites and metabolites of the tricarboxylic acid cycle (Figure 7A), cells exposed to unmodified silk fibroin nanoparticles displayed a pronounced decrease in pyruvate levels, while lactate, itaconate, and succinate increased significantly. By contrast, succinate and intracellular glucose levels decreased in cells treated with PEGylated silk fibroin nanoparticles. When comparing nanoparticle-induced changes to amino acid metabolism (Figure 7A), unmodified silk nanoparticles decreased in the intracellular levels of aspartate, glutamine, glutamate, and alanine, whereas levels of glycine, lysine, branched chain, and aromatic amino acids increased when compared to untreated controls. Interestingly, PEGylated silk fibroin nanoparticles either produced no change in some of these amino acids or caused variations in the opposite direction to that observed for unmodified particles. Energy-related metabolites were also affected by silk fibroin nanoparticle treatment (Figure 7A). When treated with unmodified particles, significant increases to creatine and phosphocreatine levels were observed at both concentrations, while only the high concentration impacted ATP levels (significant decrease). Again, milder effects were observed for macrophages treated with PEGylated silk fibroin nanoparticles. Marked changes were observed in metabolites that can act as antioxidants and/or osmoregulators or that relate to cell membrane composition (Figure 7A). In general, these metabolites showed dose-dependent decreases in cells treated with unmodified silk fibroin nanoparticles, whereas they showed increased levels in cells treated with PEGylated silk fibroin nanoparticles. Metabolites associated with lipid metabolism (Figure 7A) were also differentially affected by the two nanoparticle types, with increases in 3-hydroxybutyrate, acetate, and formate being seen only in cells treated with unmodified silk fibroin nanoparticles.

Changes in metabolic pathways were also apparent through analysis of the exometabolome (Figure 7B), with cells exposed to PEGylated silk fibroin nanoparticles consuming more glucose and excreting more lactate and itaconate when compared to untreated cells. These effects were more pronounced in cells treated with unmodified silk fibroin nanoparticles, and these cells also excreted more alanine and

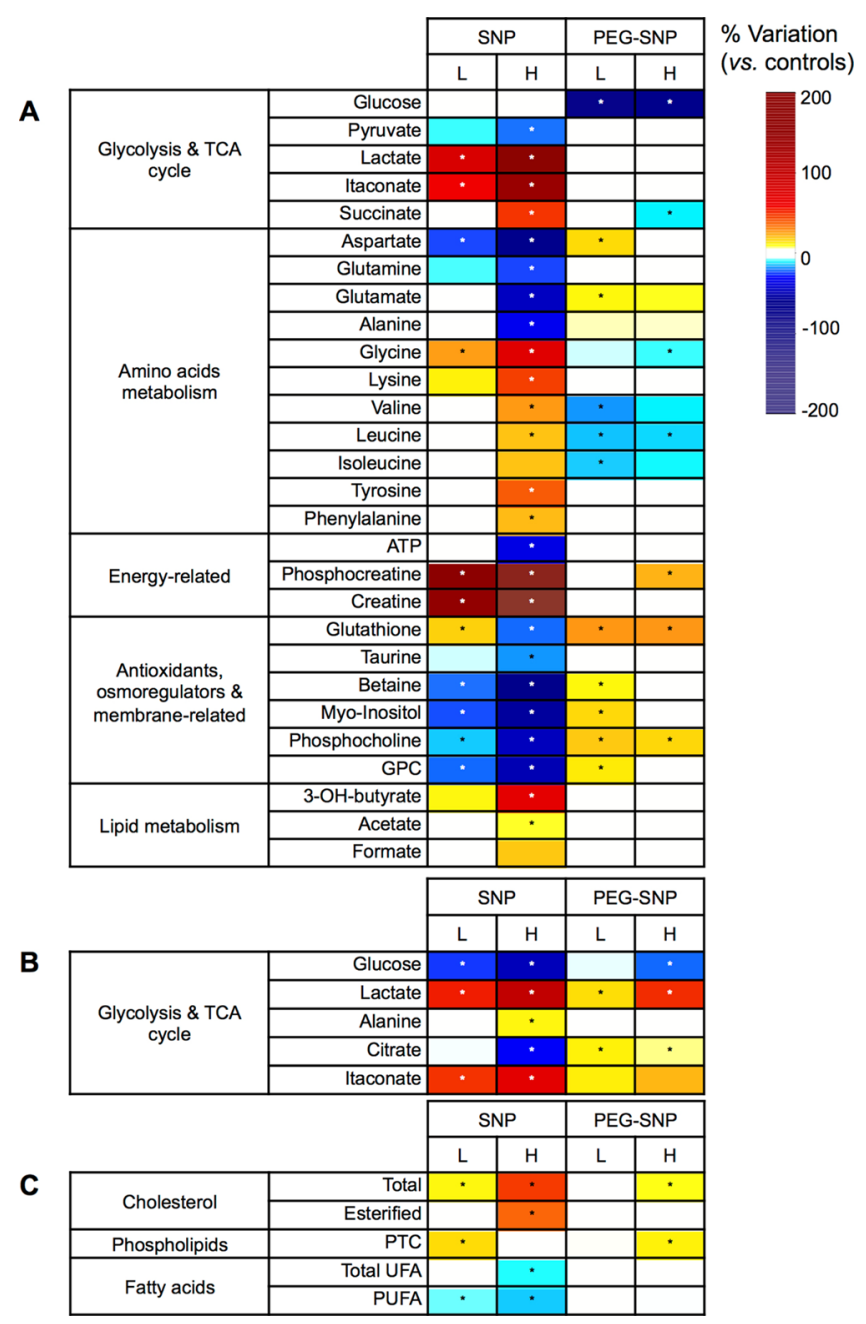

Figure 7. Heat map of the main metabolite variations in (A) aqueous extracts, (B) culture media, and (C) lipophilic extracts from macrophages exposed for $24 \mathrm{~h}$ to unmodified (SNP) or PEGylated (PEG-SNP) silk fibroin nanoparticles at concentrations of either 0.1 or $0.5 \mathrm{mg} / \mathrm{mL}$, colored according to \% variation in relation to controls. * $p$-value < 0.05. GPC: glycerophosphocholine, PTC: phosphatidylcholine, (P)UFA: (poly)unsaturated fatty acids.

less citrate when compared to untreated controls. Finally, unmodified silk fibroin nanoparticles caused increases in (total/esterified) cholesterol and phosphatidylcholine, together with decreases in unsaturated fatty acids (Figure 7C). By contrast, the effects of PEGylated silk fibroin nanoparticles were restricted to mild increases in total cholesterol and phosphatidylcholine.

\section{DISCUSSION}

The biopolymer silk has a long clinical track record in humans, ${ }^{13}$ and silk nanoparticles have recently emerged as an interesting platform for drug delivery, including anticancer drugs. ${ }^{16}$ However, the interactions of silk nanoparticles with macrophages have yet to be fully characterized, and this lack of knowledge limits our current understanding of what effects these nanoparticles will have on off-target tumor-associated macrophages. Tumor-associated macrophages show a tumorigenic profile, ${ }^{29}$ and a high distribution of these macrophages within a tumor is associated with poor patient prognosis. ${ }^{30-32}$ However, these macrophages can undergo reprogramming to 
assume a proinflammatory state that confers tumoricidal properties. ${ }^{33}$ Therefore, since anticancer nanoparticles encounter macrophages upon accessing a tumor site, they provide a unique opportunity to modulate the inflammatory profiles of tumor-associated macrophages and thereby maximize the intratumoral performance. ${ }^{34-36}$ The aim of the present study was to examine the baseline performances of both unmodified and PEGylated silk fibroin nanoparticles by monitoring the effects of particle PEGylation and concentration on the inflammatory phenotype and metabolic profiles of macrophages. We have opted to study the response of silk fibroin nanoparticles without an anticancer drug payload to simplify data interpretation and to eliminate drug-mediated cofounding effects (e.g., drug-induced cytotoxicity).

Similar to our previous reports ${ }^{17}$ we found that PEGylation of silk fibroin nanoparticles increased the hydrodynamic radius and moderated the surface charge when compared to unmodified nanoparticles (Figure 1B); an observation that aligns well with the literature (reviewed by Rabanel et al.). ${ }^{37}$ We now provide additional evidence that the interaction of either unmodified or PEGylated silk fibroin nanoparticles with the macrophage plasma membrane caused no damage to the cell membrane (Figure 2B). As we have observed previously, ${ }^{17,38}$ the unmodified silk nanoparticles had a higher propensity to aggregate in physiological media when compared to the PEGylated silk fibroin nanoparticles (Figure 2C). However, this aggregation did not affect the macrophages' ability to phagocytose and traffic unmodified silk fibroin particles into lysosomes (Figure 3A,B). The somewhat larger appearance of the lysosomes containing unmodified silk fibroin nanoparticles, when compared with those containing PEGylated silk fibroin nanoparticles, might be a consequence of the larger size of the internalized nanoparticle aggregates.

Both silk fibroin nanoparticle formulations were internalized and trafficked into lysosomes, but the downstream effects on the inflammatory profile of the macrophages were dependent on the surface modification and particle concentration. A high concentration of unmodified silk fibroin nanoparticles primed the RAW 264.7 macrophages toward a proinflammatory phenotype, as evidenced by significant increases in levels of classical proinflammatory mediators (e.g., TNF- $\alpha$, nitric oxide, Figure $3 \mathrm{~A}, \mathrm{~B}){ }^{39-41}$ enhanced total antioxidant activity, and reduction in levels of reactive oxygen species (Figure 3C,D). Reactive oxygen species levels are implicated in the differentiation of macrophages into wound-healing and tumorassociated phenotypes. ${ }^{42}$ However, even at the low nanoparticle concentration, unmodified silk fibroin nanoparticles were able to induce a proinflammatory shift in the macrophage phenotype, although to a lesser extent than the shift observed at high concentrations (i.e., less production of nitric oxide and reactive oxygen species, but similar promotion of TNF- $\alpha$ and antioxidant levels when compared to the high nanoparticle dose).

The biological significance of macrophage priming at the high silk fibroin nanoparticle dose was also evident by the observed upregulation of the macrophage cytokine profile (e.g., colony stimulating factors, chemokines, and other essential proinflammatory substances) (Figure 3C). However, PEGylation apparently modulated the immunomodulatory effects of silk fibroin nanoparticles. At the high nanoparticle concentration, PEGylation moderated the production of proinflammatory mediators, including TNF- $\alpha$, nitric oxide, total antioxidant activity, and reactive oxygen species when compared to the response to unmodified silk fibroin nanoparticles (Figure 4). At the high concentration, the PEGylated silk fibroin nanoparticles only evoked subtle differences in cytokine production between the treated and control cells (Figure 4D). Overall, these observations are important because they suggest the possibility of differential priming of macrophages, even at high nanoparticle concentrations.

While nanoparticles for drug delivery are typically designed to evade the immune system, a proimmunogenic phenotype could be exploited to maximize intratumoral performance. ${ }^{34,35}$ We and others have shown a link between metabolic reprogramming and cell behavior, such as macrophage activation status. ${ }^{43}$ We therefore examined the baseline performances of both unmodified and PEGylated silk nanoparticles by monitoring nanoparticle-induced metabolic changes. Exposure to silk fibroin nanoparticles affected macrophage metabolism in a nanoparticle-type- and concentration-dependent fashion (Figure 6). In agreement with our previous study ${ }^{12}$ and similar to the metabolic phenotype described for lipopolysaccharide-activated proinflammatory macrophages, ${ }^{44,45}$ cells treated with unmodified silk nanoparticles increased cellular glycolytic activity, as indicated by significantly increased glucose consumption, upregulated pyruvate to lactate conversion, and increased lactate and alanine excretion. Increased glycolysis was also apparent in cells treated with PEGylated nanoparticles, although the lactate levels in this case were only elevated in the medium supernatants, not intracellularly.

Cells treated with unmodified silk fibroin nanoparticles further displayed enhanced production of succinate and itaconate, which is consistent with the reprogramming of the tricarboxylic acid cycle associated with a proinflammatory state. $^{46}$ Succinate is known to inhibit cytosolic prolyl hydroxylases, thereby stabilizing $\operatorname{HIF} 1 \alpha$, whose target genes include glycolytic enzymes and inflammatory factors. ${ }^{47}$ Additionally, succinate oxidation by succinate dehydrogenase drives the production of reactive oxygen species. ${ }^{48}$ By contrast, itaconate, which is produced from citrate via cis-aconitate, ${ }^{49}$ inhibits succinate dehydrogenase $\mathrm{e}^{50}$ and acts as a counterbalance to limit inflammation. ${ }^{51,52}$ This metabolite inhibits succinate dehydrogenase and activates the anti-inflammatory and antioxidant transcription factor $\mathrm{Nrf} 2$, thus restricting downstream inflammatory gene expression and limiting the production of proinflammatory mediators like IL- $1 \beta$ and IL$6 .{ }^{52}$ Activation of the $\mathrm{Nrf} 2$ /antioxidant response element pathway could result in increased expression of antioxidant enzymes (e.g., catalase, superoxide dismutase), as well as increased glutathione synthesis. ${ }^{53}$ Therefore the observed itaconate increase and enhanced antioxidant capacity could ultimately equip cells better to neutralize reactive oxygen species. This proposal is consistent with the observed decrease in reactive oxygen species upon nanoparticle treatment. Therefore, our results corroborate a close link between rewiring of the tricarboxylic acid cycle, the cell redox status, and the production of proinflammatory cytokines. ${ }^{53}$ Another consequence of this type of metabolic reprogramming could be the observed ATP depletion in cells exposed to a high concentration of unmodified silk fibroin nanoparticles, which, as noted in our previous study, ${ }^{12}$ could be due to both inhibition of succinate dehydrogenase (and hence oxidative phosphorylation $)^{54}$ and downregulation of substrate-level phosphorylation. $^{55}$ 
The marked decrease in intracellular levels of some amino acids in cells treated with unmodified silk fibroin nanoparticles further supports tricarboxylic acid cycle reprogramming. In particular, glutamine/glutamate and aspartate could be used to replenish $\alpha$-ketoglutarate and oxaloacetate, respectively, and so could be involved in the aspartate-arginosuccinate shunt, which is important in lipopolysaccharide-induced macrophage activation. $^{45}$ By contrast, a different set of amino acids (glycine, lysine, branched chain, and aromatic amino acids) increased in cells treated with unmodified silk nanoparticles, possibly reflecting protein catabolism. ${ }^{12}$ The PEGylated silk fibroin nanoparticles did not have a similar effect on the tricarboxylic acid cycle. No intracellular increases were noted in succinate or itaconate upon nanoparticle treatment although succinate levels were decreased at the higher nanoparticle dose. Clear reduction in tricarboxylic acid cycle rewiring and itaconate production coincided with a reduced inflammatory phenotype in RAW 264.7 macrophages, and this supports observations made with unmodified silk fibroin nanoparticles.

Another prominent effect of unmodified silk fibroin nanoparticles was the significant intracellular upregulation of creatine and phosphocreatine. Although the functional relevance of these metabolites in macrophage responses to nanoparticles remains to be determined, one possibility is that the creatine kinase/phosphocreatine system plays an important role, possibly in relation to energy buffering and phagocytic function. ${ }^{56}$ Interestingly, PEGylated silk fibroin nanoparticles produced a milder increase in phosphocreatine and no change in creatine levels, suggesting a less extensive impact on the creatine kinase system. One other explanation for the increased lysosome size observed in response to unmodified silk fibroin nanoparticles might be a differential demand on the creatine kinase system of the phagocytosing macrophages (Figures 2 and 3). The lower aggregation of PEGylated silk fibroin nanoparticles could possibly place less demand on the creatine kinase system. This observation warrants further investigation.

The levels of several metabolites involved in osmoregulation, antioxidant protection, and/or cell membrane composition also showed distinct variation patterns in cells exposed to unmodified or PEGylated silk fibroin nanoparticles. In response to unmodified silk fibroin nanoparticles the levels of antioxidant metabolites glutathione (albeit increased at the lower dose treatment), taurine, and betaine decreased, which may possibly reflect their use to counterbalance reactive oxygen species generation by proinflammatory macrophages. $^{57,58}$ The observed decrease in reactive oxygen species levels in nanoparticle-treated cells is in line with these observations. By contrast, the levels of glutathione and betaine, to a lesser extent, increased in cells treated with PEGylated silk nanoparticles. Opposite variations were also found for myoinositol, phosphocholine, and glycerophosphocholine, which are metabolites with known involvement in membrane remodeling and osmotic balance.

Last, unmodified silk fibroin nanoparticles, but not PEGylated ones, caused increases in metabolites that could arise from enhanced lipid metabolism: 3-hydroxybutyrate, acetate, and formate. Changes in cellular lipid composition were also apparent from the analysis of lipophilic extracts (Figure 6C). Treatment with unmodified silk fibroin nanoparticles and, to a lesser extent, PEGylated particles caused cells to increase their cholesterol and phospholipid content. Additionally, the levels of unsaturated fatty acids decreased upon exposure to unmodified nanoparticles, again possibly reflecting remodeling of membrane composition and fluidity.

\section{CONCLUSIONS}

In summary, we have demonstrated that unmodified-and, to a lesser extent, PEGylated-silk fibroin nanoparticles modulated macrophage activation and metabolism in a concentration-dependent and particle-type-dependent manner. Specifically, unmodified nanoparticles induced a proinflammatory phenotype that was characterized by increased cytokine release, enhanced nitric oxide production, and elevated antioxidant levels, as well as decreased reactive oxygen species levels. Extensive rewiring of the metabolic profiles, including glycolysis, the tricarboxylic acid cycle, and amino acid and antioxidant metabolite biosynthesis, as well as creatine kinase system activity, was also observed. This ability to modulate the macrophage phenotype could be especially useful in the context of anticancer drug delivery, as silk fibroin nanoparticles would act both as carriers for chemotherapeutics and as synergistic attenuators of tumor-associated macrophages toward a more tumoricidal phenotype.

\section{ASSOCIATED CONTENT}

\section{S Supporting Information}

The Supporting Information is available free of charge on the ACS Publications website at DOI: 10.1021/acsami.8b18716.

Positive and negative controls used to calculate mean cell viability in MTT cytotoxicity assays $(n=3)$; in vitro cytotoxicity, determined by MTT, of PEG following a 24 $\mathrm{h}$ incubation in complete media supplemented with $0-$ $1000 \mu \mathrm{g} / \mathrm{mL}$ nanoparticles $(n=3)$ (PDF)

\section{AUTHOR INFORMATION}

\section{Corresponding Authors}

*E-mail: ioladuarte@ua.pt. Tel: +351 234401418 (I.F.D.).

*E-mail: philipp.seib@strath.ac.uk.philipp.seib@SeibLab.com. Tel: +44 (0) 1415482510 (F.P.S.).

\section{ORCID}

Iola F. Duarte: 0000-0003-4289-9256

F. Philipp Seib: 0000-0002-1955-1975

\section{Author Contributions}

J.C. conducted NMR data acquisition and processing, T.W. conducted silk nanoparticle production and characterization using SEM imaging. All cell cultures were conducted by J.D.T. All authors (J.D.T., T.W., J.C., I.F.D., and F.P.S.) designed the research, discussed the results, and/or advised on the analysis. J.D.T. wrote the manuscript with support from the other authors. I.F.D. and F.P.S. conceived the study.

\section{Notes}

The authors declare no competing financial interest. All data created during this research are openly available from the University of Strathclyde-Pure at https://doi.org/10. 15129/f03c40f2-1eef-44a0-891a-628abbe5cb16.

\section{ACKNOWLEDGMENTS}

The University of Strathclyde supported this study with a Research and Development Grant 1715 (F.P.S, I.F.D.). J.D.T.'s Ph.D. studentship is sponsored by the EPSRC Doctoral Training Partnership (EP/M508159/1), University of Strathclyde. The authors thank the International Strategic Partnership between the University of Strathclyde and Nanyang 
Technological University, Singapore for funding Ph.D. candidate T.W. The work was also developed in the scope of the project CICECO-Aveiro Institute of Materials, FCT Ref. UID/CTM/50011/2019, financed by national funds through the FCT/MCTES. The authors also acknowledge the Portuguese National NMR (PTNMR) Network, supported with FCT funds, and the European Union Framework Programme for Research and Innovation HORIZON 2020, under the TEAMING Grant agreement No 739572 - The Discoveries CTR. I.F.D. further acknowledges FCT/MCTES for a research contract under the Program "Investigador FCT" 2014.

\section{REFERENCES}

(1) Nakamura, H.; Jun, F.; Maeda, H. Development of NextGeneration Macromolecular Drugs Based on the EPR Effect: Challenges and Pitfalls. Expert Opin. Drug Delivery 2014, 12, 53-64.

(2) Srinivasarao, M.; Galliford, C. V.; Low, P. S. Principles in the Design of Ligand-Targeted Cancer Therapeutics and Imaging Agents. Nat. Rev. Drug Discovery 2015, 14, 203-219.

(3) Yao, V. J.; D’Angelo, S.; Butler, K. S.; Theron, C.; Smith, T. L.; Marchiò, S.; Gelovani, J. G.; Sidman, R. L.; Dobroff, A. S.; Brinker, C. J.; Bradbury, A. R. M.; Arap, W.; Pasqualini, R. Ligand-Targeted Theranostic Nanomedicines against Cancer. J. Controlled Release 2016, 240, 267-286.

(4) Shi, J.; Kantoff, P. W.; Wooster, R.; Farokhzad, O. C. Cancer Nanomedicine: Progress, Challenges and Opportunities. Nat. Rev. Cancer 2017, 17, 20-37.

(5) Nel, A. E.; Mädler, L.; Velegol, D.; Xia, T.; Hoek, E. M. V.; Somasundaran, P.; Klaessig, F.; Castranova, V.; Thompson, M. Understanding Biophysicochemical Interactions at the Nano-Bio Interface. Nat. Mater. 2009, 8, 543-557.

(6) Tenzer, S.; Docter, D.; Kuharev, J.; Musyanovych, A.; Fetz, V.; Hecht, R.; Schlenk, F.; Fischer, D.; Kiouptsi, K.; Reinhardt, C.; Landfester, K.; Schild, H.; Maskos, M.; Knauer, S. K.; Stauber, R. H. Rapid Formation of Plasma Protein Corona Critically Affects Nanoparticle Pathophysiology. Nat. Nanotechnol. 2013, 8, 772.

(7) Blanco, E.; Shen, H.; Ferrari, M. Principles of Nanoparticle Design for Overcoming Biological Barriers to Drug Delivery. Nat. Biotechnol. 2015, 33, 941-951.

(8) Harris, J. M.; Chess, R. B. Effect of Pegylation on Pharmaceuticals. Nat. Rev. Drug Discovery 2003, 2, 214-221.

(9) Mosser, D. M.; Edwards, J. P. Exploring the Full Spectrum of Macrophage Activation. Nat. Rev. Immunol. 2008, 8, 958-969.

(10) Dobrovolskaia, M. A.; McNeil, S. E. Immunological Properties of Engineered Nanomaterials. Nat. Nanotechnol. 2007, 2, 469-478.

(11) Wolfram, J.; Zhu, M.; Yang, Y.; Shen, J.; Gentile, E.; Paolino, D.; Fresta, M.; Nie, G.; Chen, C.; Shen, H.; Ferrari, M.; Zhao, Y. Safety of Nanoparticles in Medicine. Curr. Drug Targets 2015, 16, 1671-1681.

(12) Saborano, R.; Wongpinyochit, T.; Totten, J. D.; Johnston, B. F.; Seib, F. P.; Duarte, I. F. Metabolic Reprogramming of Macrophages Exposed to Silk, Poly(Lactic-Co-Glycolic Acid), and Silica Nanoparticles. Adv. Healthcare Mater. 2017, 6, 1601240.

(13) Holland, C.; Numata, K.; Rnjak-Kovacina, J.; Seib, F. P. The Biomedical Use of Silk: Past, Present, Future. Adv. Healthcare Mater. 2019, 1800465.

(14) Seib, F. P.; Maitz, M. F.; Hu, X.; Werner, C.; Kaplan, D. L. Impact of Processing Parameters on the Haemocompatibility of Bombyx Mori Silk Films. Biomaterials 2012, 33, 1017-1023.

(15) Wongpinyochit, T.; Johnston, B. F.; Seib, F. P. Manufacture and Drug Delivery Applications of Silk Nanoparticles. J. Visualized Exp. 2016, 54669.

(16) Seib, F. P. Silk Nanoparticles-an Emerging Anticancer Nanomedicine. AIMS Bioeng. 2017, 4, 239-258.

(17) Wongpinyochit, T.; Uhlmann, P.; Urquhart, A. J.; Seib, F. P. PEGylated Silk Nanoparticles for Anticancer Drug Delivery. Biomacromolecules 2015, 150929190037005.
(18) Wongpinyochit, T.; Johnston, B. F.; Seib, F. P. Degradation Behavior of Silk Nanoparticles - Enzyme Responsiveness. ACS Biomater. Sci. Eng. 2018, 4, 942-951.

(19) Totten, J. D.; Wongpinyochit, T.; Seib, F. P. Silk Nanoparticles: Proof of Lysosomotropic Anticancer Drug Delivery at Single-Cell Resolution. J. Drug Targeting 2017, 25, 865-872.

(20) Seib, F. P.; Jones, G. T.; Rnjak-Kovacina, J.; Lin, Y.; Kaplan, D. L. PH-Dependent Anticancer Drug Release from Silk Nanoparticles. Adv. Healthcare Mater. 2013, 2, 1606-1611.

(21) Raschke, W. C.; Baird, S.; Ralph, P.; Nakoinz, I. Functional Macrophage Cell Lines Transformed by Abelson Leukemia Virus. Cell 1978, 15, 261-267.

(22) Hu, X.; Kaplan, D.; Cebe, P. Determining Beta-Sheet Crystallinity in Fibrous Proteins by Thermal Analysis and Infrared Spectroscopy. Macromolecules 2006, 39, 6161-6170.

(23) Manders, E. M. M.; Verbeek, F. J.; Aten, J. A. Measurement of Co-Localization of Objects in Dual-Colour Confocal Images. J. Microsc. 1993, 169, 375-382.

(24) Dunn, K. W.; Kamocka, M. M.; McDonald, J. H. A Practical Guide to Evaluating Colocalization in Biological Microscopy. AJP Cell Physiol. 2011, 300, C723-C742.

(25) Sgouras, D.; Duncan, R. Methods for the Evaluation of Biocompatibility of Soluble Synthetic Polymers Which Have Potential for Biomedical Use: 1 - Use of the Tetrazolium-Based Colorimetric Assay (MTT) as a Preliminary Screen for Evaluation ofin Vitro Cytotoxicity. J. Mater. Sci. Mater. Med. 1990, 1, 61-68.

(26) Misko, T. P.; Schilling, R. J.; Salvemini, D.; Moore, W. M.; Currie, M. G. A Fluorometric Assay for the Measurement of Nitrite in Biological Samples. Anal. Biochem. 1993, 11-16.

(27) Ridnour, L. A.; Sim, J. E.; Hayward, M. A.; Wink, D. A.; Martin, S. M.; Buettner, G. R.; Spitz, D. R. A Spectrophotometric Method for the Direct Detection and Quantitation of Nitric Oxide, Nitrite, and Nitrate in Cell Culture Media. Anal. Biochem. 2000, 281, 223-229.

(28) Berben, L.; Sereika, S. M.; Engberg, S. Effect Size Estimation: Methods and Examples. Int. J. Nurs. Stud. 2012, 49, 1039-1047.

(29) Mantovani, A.; Sozzani, S.; Locati, M.; Allavena, P.; Sica, A. Macrophage Polarization: Tumor-Associated Macrophages as a Paradigm for Polarized M2 Mononuclear Phagocytes. Trends Immunol. 2002, 23, 549-555.

(30) Lewis, C. E.; Pollard, J. W. Distinct Role of Macrophages in Different Tumor Microenvironments. Cancer Res. 2006, 66, 605-612.

(31) De Palma, M.; Lewis, C. E. Macrophage Regulation of Tumor Responses to Anticancer Therapies. Cancer Cell 2013, 23, 277-286.

(32) Zhang, Q.; Liu, L.; Gong, C.; Shi, H.; Zeng, Y.; Wang, X.; Zhao, Y.; Wei, Y. Prognostic Significance of Tumor-Associated Macrophages in Solid Tumor: A Meta-Analysis of the Literature. PLoS One 2012, 7, No. e50946.

(33) Mantovani, A.; Marchesi, F.; Malesci, A.; Laghi, L.; Allavena, P. Tumour-Associated Macrophages as Treatment Targets in Oncology. Nat. Rev. Clin. Oncol. 2017, 14, 399.

(34) Ostuni, R.; Kratochvill, F.; Murray, P. J.; Natoli, G. Macrophages and Cancer: From Mechanisms to Therapeutic Implications. Trends Immunol. 2015, 36, 229-239.

(35) Ngambenjawong, C.; Gustafson, H. H.; Pun, S. H. Progress in Tumor-Associated Macrophage (TAM)-Targeted Therapeutics. Adv. Drug Deliv Rev. 2017, 114, 206-221.

(36) Singh, Y.; Pawar, V. K.; Meher, J. G.; Raval, K.; Kumar, A.; Shrivastava, R.; Bhadauria, S.; Chourasia, M. K. Targeting Tumor Associated Macrophages (TAMs) via Nanocarriers. J. Controlled Release 2017, 254, 92-106.

(37) Rabanel, J. M.; Hildgen, P.; Banquy, X. Assessment of PEG on Polymeric Particles Surface, a Key Step in Drug Carrier Translation. J. Controlled Release 2014, 185, 71-87.

(38) Maitz, M. F.; Sperling, C.; Wongpinyochit, T.; Herklotz, M.; Werner, C.; Seib, F. P. Biocompatibility Assessment of Silk Nanoparticles: Hemocompatibility and Internalization by Human Blood Cells. Nanomedicine Nanotechnology, Biol. Med. 2017, 13, $2633-2642$ 
(39) Griffin, G. K.; Newton, G.; Tarrio, M. L.; Bu, D.; MagantoGarcia, E.; Azcutia, V.; Alcaide, P.; Grabie, N.; Luscinskas, F. W.; Croce, K. J.; Lichtman, A. H. IL-17 and TNF- $\alpha$ Sustain Neutrophil Recruitment during Inflammation through Synergistic Effects on Endothelial Activation. J. Immunol. 2012, 188, 6287-6299.

(40) Bogdan, C. Nitric Oxide Synthase in Innate and Adaptive Immunity: An Update. Trends Immunol. 2015, 36, 161-178.

(41) MacMicking, J.; Xie, Q.; Nathan, C. Nitric Oxide and Macrophage Function. Annu. Rev. Immunol. 1997, 15, 323-350.

(42) Zhang, Y.; Choksi, S.; Chen, K.; Pobezinskaya, Y.; Linnoila, I.; Liu, Z.-G. ROS Play a Critical Role in the Differentiation of Alternatively Activated Macrophages and the Occurrence of TumorAssociated Macrophages. Cell Res. 2013, 23, 898.

(43) Van den Bossche, J.; O’Neill, L. A.; Menon, D. Macrophage Immunometabolism: Where Are We (Going)? Trends Immunol. 2017, 38, 395-406.

(44) Rodríguez-Prados, J.-C.; Través, P. G.; Cuenca, J.; Rico, D.; Aragonés, J.; Martín-Sanz, P.; Cascante, M.; Boscá, L. Substrate Fate in Activated Macrophages: A Comparison between Innate, Classic, and Alternative Activation. J. Immunol. 2010, 185, 605-614.

(45) Jha, A. K.; Huang, S. C.-C.; Sergushichev, A.; Lampropoulou, V.; Ivanova, Y.; Loginicheva, E.; Chmielewski, K.; Stewart, K. M.; Ashall, J.; Everts, B.; Pearce, E. J.; Driggers, E. M.; Artyomov, M. N. Network Integration of Parallel Metabolic and Transcriptional Data Reveals Metabolic Modules That Regulate Macrophage Polarization. Immunity 2015, 42, 419-430.

(46) Ryan, D. G.; O’Neill, L. A. J. Krebs Cycle Rewired for Macrophage and Dendritic Cell Effector Functions. FEBS Lett. 2017, 591, 2992-3006.

(47) Tannahill, G. M.; Curtis, A. M.; Adamik, J.; PalssonMcDermott, E. M.; McGettrick, A. F.; Goel, G.; Frezza, C.; Bernard, N. J.; Kelly, B.; Foley, N. H.; Zheng, L.; Gardet, A.; Tong, Z.; Jany, S. S.; Corr, S. C.; Haneklaus, M.; Caffrey, B. E.; Pierce, K.; Walmsley, S.; Beasley, F. C.; Cummins, E.; Nizet, V.; Whyte, M.; Taylor, C. T.; Lin, H.; Masters, S. L.; Gottlieb, E.; Kelly, V. P.; Clish, C.; Auron, P. E.; Xavier, R. J.; O’Neill, L. A. J. Succinate Is an Inflammatory Signal That Induces IL- $1 \beta$ through HIF-1 $\alpha$. Nature 2013, 496, 238-242.

(48) Mills, E. L.; Kelly, B.; Logan, A.; Costa, A. S. H.; Varma, M.; Bryant, C. E.; Tourlomousis, P.; Däbritz, J. H. M.; Gottlieb, E.; Latorre, I.; Corr, S. C.; McManus, G.; Ryan, D.; Jacobs, H. T.; Szibor, M.; Xavier, R. J.; Braun, T.; Frezza, C.; Murphy, M. P.; O’Neill, L. A. Succinate Dehydrogenase Supports Metabolic Repurposing of Mitochondria to Drive Inflammatory Macrophages. Cell 2016, 167, 457-470.

(49) Michelucci, A.; Cordes, T.; Ghelfi, J.; Pailot, A.; Reiling, N.; Goldmann, O.; Binz, T.; Wegner, A.; Tallam, A.; Rausell, A.; Buttini, M.; Linster, C. L.; Medina, E.; Balling, R.; Hiller, K. ImmuneResponsive Gene 1 Protein Links Metabolism to Immunity by Catalyzing Itaconic Acid Production. Proc. Natl. Acad. Sci. U. S. A. 2013, 110, 7820-7825.

(50) Cordes, T.; Wallace, M.; Michelucci, A.; Divakaruni, A. S.; Sapcariu, S. C.; Sousa, C.; Koseki, H.; Cabrales, P.; Murphy, A. N.; Hiller, K.; Metallo, C. M. Immunoresponsive Gene 1 and Itaconate Inhibit Succinate Dehydrogenase to Modulate Intracellular Succinate Levels. J. Biol. Chem. 2016, 291, 14274-14284.

(51) Lampropoulou, V.; Sergushichev, A.; Bambouskova, M.; Nair, S.; Vincent, E. E.; Loginicheva, E.; Cervantes-Barragan, L.; Ma, X.; Huang, S. C.-C.; Griss, T.; Weinheimer, C. J.; Khader, S.; Randolph, G. J. J.; Pearce, E. J.; Jones, R. G.; Diwan, A.; Diamond, M. S.; Artyomov, M. N. Itaconate Links Inhibition of Succinate Dehydrogenase with Macrophage Metabolic Remodeling and Regulation of Inflammation. Cell Metab. 2016, 24, 158-166.

(52) Mills, E. L.; Ryan, D. G.; Prag, H. A.; Dikovskaya, D.; Menon, D.; Zaslona, Z.; Jedrychowski, M. P.; Costa, A. S. H.; Higgins, M.; Hams, E.; Szpyt, J.; Runtsch, M. C.; King, M. S.; McGouran, J. F.; Fischer, R.; Kessler, B. M.; McGettrick, A. F.; Hughes, M. M.; Carroll, R. G.; Booty, L. M.; Knatko, E. V.; Meakin, P. J.; Ashford, M. L. J.; Modis, L. K.; Brunori, G.; Sévin, D. C.; Fallon, P. G.; Caldwell, S. T.;
Kunji, E. R. S.; Chouchani, E. T.; Frezza, C.; Dinkova-Kostova, A. T.; Hartley, R. C.; Murphy, M. P.; O’Neill, L. A. Itaconate Is an AntiInflammatory Metabolite That Activates Nrf2 via Alkylation of KEAP1. Nature 2018, 556, 113-117.

(53) Schieber, M.; Chandel, N. S. ROS Function in Redox Signaling and Oxidative Stress. Curr. Biol. 2014, 24, R453-R462.

(54) EL Kasmi, K. C.; Stenmark, K. R. Contribution of Metabolic Reprogramming to Macrophage Plasticity and Function. Semin. Immunol. 2015, 27, 267-275.

(55) Németh, B.; Doczi, J.; Csete, D.; Kacso, G.; Ravasz, D.; Adams, D.; Kiss, G.; Nagy, A. M.; Horvath, G.; Tretter, L.; Mócsai, A.; Csépányi-Kömi, R.; Iordanov, I.; Adam-Vizi, V.; Chinopoulos, C. Abolition of Mitochondrial Substrate-Level Phosphorylation by Itaconic Acid Produced by LPS-Induced Irg1 Expression in Cells of Murine Macrophage Lineage. FASEB J. 2016, 30, 286-300.

(56) Loike, J. D.; Somes, M.; Silverstein, S. C. Creatine Uptake, Metabolism, and Efflux in Human Monocytes and Macrophages. Am. J. Physiol: Cell Physiol. 1986, 251, C128-35.

(57) Marcinkiewicz, J.; Grabowska, A.; Bereta, J.; Stelmaszynska, T. Taurine Chloramine, a Product of Activated Neutrophils, Inhibits in Vitro the Generation of Nitric Oxide and Other Macrophage Inflammatory Mediators. J. Leukoc Biol. 1995, 58, 667-674.

(58) Murray, P. J.; Wynn, T. A. Protective and Pathogenic Functions of Macrophage Subsets. Nat. Rev. Immunol. 2011, 11, 723-737. 